\title{
ED Visits Related to Marijuana Exposures in the Denver Metropolitan Area of Colorado
}

\author{
Yushiuan Chen*1, Michele Askenazi', Kathryn H. DeYoung², Bernadette Albanese', \\ Lourdes W. Yun ${ }^{2}$ and Todd Hockenberry ${ }^{1}$
}

${ }^{1}$ Emergency Preparedness and Response, Tri-County Health Department, Greenwood Village, CO, USA; ${ }^{2}$ Denver Public Health, Denver, CO, USA

\section{Objective}

Adverse health effects related to marijuana (MJ) use may disproportionately impact populations based on age or gender. To explore whether disparities exist among persons seeking emergency department (ED) care related to MJ use, Tri-County Health Department (TCHD) and Denver Public Health (DPH) developed MJ use case definitions, described patient demographics, mapped patients' geographic distribution relative to marijuana dispensary locations, evaluated access to healthcare, and investigated the potential impact of $\mathrm{MJ}$ on pediatric health.

\section{Introduction}

Assessing health disparities and access to healthcare has been an important issue for emergency preparedness and response efforts in the Denver metropolitan area. There have been several high profile MJ-related illness outbreaks in the US over the past 2 years. The legalization and retail sale of recreational $\mathrm{MJ}$ in Colorado necessitates enhanced surveillance for adverse effects from MJ use. TCHD and DPH coordinated to use syndromic surveillance data to provide situational awareness and timely outbreak detection related to MJ, including health disparities and overall impacts on population health.

\section{Methods}

We used syndromic surveillance data from ED visits to 15 hospitals in Adams, Arapahoe, Denver, and Douglas counties; census poverty data; education and school data from Colorado; and locations of medical and recreational MJ dispensaries. An MJ-related case (MJC) was defined as a case where text in the chief complaint, provider's diagnosis, and/or diagnosis ICD9 code contained terms including "Marijuana," "MJ," "Cannabis," "Edible," "E854.1," "304.3," "969.6," and "305.2."

We used the Electronic Surveillance System for the Early Notification of Community Based Epidemic (ESSENCE) to identify and evaluate MJ cases and created maps in ArcGIS to illustrate the distribution of pediatric and adult MJC relative to MJ dispensaries and healthcare facilities. The patient identifier number was used for deduplication and provider's diagnosis, chief complaint, and diagnosis code were verified accordingly. Access to healthcare facilities was assessed by comparing aggregated patient zip code and county code information to ED locations.

All MJCs were included and the demographic attributes of MJCs were analyzed after a manual review and de-duplication process. After October 2015, ICD-10 codes will be employed and adjustments will be made to examine the MJ search terms.

\section{Results}

After two additional hospitals began reporting data in August 2015, the total number of monthly ED visits increased $23.9 \%$ for a total of 85,357 visits. There were 279 (3.3\%) MJCs identified. By age group, persons $18-44$ years represented $54 \%$ of MJCs as compared to persons aged 5-17 years (22.3\%), 45-64 years $(21.7 \%)$, and 65 years or older $(1.9 \%)$. A higher percentage of MJCs were males $(66.2 \%)$. MJCs were residents in Denver County (43\%), Adams
(15\%), Arapahoe (15\%) and Douglas (3\%) counties, and Denver and Arapahoe Counties had a higher percentage of pediatric MJCs (7.29\% and $4.58 \%$, respectively) than the other two counties. Ongoing MJC analyses, including patients' proximity to healthcare facilities and MJ dispensaries and impacts on school-aged patients, will be presented in December.

\section{Conclusions}

Preliminary results provide an overview of the prevalence of ED visits related to MJ use and identify differences in MJCs by age, gender, and geographic location. Males and persons aged 18 to 44 years constituted a higher proportion of MJCs, which may be related to differences in usage patterns as identified by population health surveys such as the Behavioral Risk Factor Surveillance System (BRFSS). There were also geographic disparities, with Denver and Arapahoe Counties having a higher percentage of pediatric MJ cases. Additionally, Denver County had the most recreational marijuana dispensaries. More advanced spatial analysis will describe any association between distribution of MJ dispensaries and pediatric cases.

The study identified limitations in using syndromic surveillance data for this purpose, including the lack of a standardized case definition or ICD-9 code to identify for MJCs. Additionally, MJrelated health outcomes might be underreported in ICD-9 codes. TCHD and DPH will continue monitoring syndromic surveillance data quality for a 180-day baseline period and add ICD-10 codes to the search criteria to create more specific case definitions.

Keywords

Syndromic Surveillance; Marijuana; ESSENCE; Cannabis

\section{*Yushiuan Chen}

E-mail: ychen@tchd.org 